DIRETOR DA FACULDADE DE CIÊNCIAS ECONÓMICAS:

Prof. Antônio Carlos Santos Rosa

VICE-DIRETOR: Prof. Nelson Rokembach

CHEFE DO DEPARTAMENTO DE CIENNCIAS ECONÓMICAS:

Prof. Renato Batista Masina

CONSELHO EDITORIAL: Prof. Pedro Cezar Dutra Fonseca (Presidente)

Prof. Achyles Barcelos da Costa

Prof. Carlos Augusto Crusius

Prof. Claudio Francisco Accurso

Prof. Edgar Augusto Lanzer

Prof. Ernani Hickmann

Prof. Nali de Jesus de Souza

Prof. Nuno Renan L. de Figueiredo Pinto

Profa Otilia Beatriz Kroeff Carrion

Prof. Roberto Camps Moraes

Profa Yeda Rorato Crusius

ANÁLISE ECONOMICA é uma publicação semestral da Faculdade de Ciências Econômicas da Universidade Federal do Rio Grande do Sul, visando divulgar estudos e pesquisas de seu corpo docente e discente na área de Economia. Aceitam-se, entretanto, artigos e resenhas bibliográficas de economistas e técnicos não vinculados à Instituição. As matérias assinadas são de responsabilidade exclusiva dos autores. É permitida a reprodução parcial para fins didáticos.

Toda a correspondência, material para publicação, assinaturas e permutas devem ser dirigidas a:

Prof. PEDRO CEZAR DUTRA FONSECA

Revista Anál ise Econômica

Avenida João Pessoa, $52-3^{\circ}$ andar

90.000 - Porto Alegre (RS) - Brasil 


\section{CRESCIMENTO ABSOLUTA E RELATIVAMENTE EMPOBRECEDOR E A TESE DE PREBISCH E SINGER *}

Andrea Maneschi ***

Initrodução

O propósito deste artigo é analisar as implicações do crescimento econômico sobre os níveis absolutos e relativos de renda real per capita em dois países que comerciam entre si. Nos anos 50, Johnson $(1953,1955)$ e Bhagwati (1958) elucidaram as condições restritivas sob as quais um pais pode incorrer no "crescimento empobrecedor", decorrente de um movimento adverso nos seus termos de intercâmbio que mais do que compensa o seu crescimento de produto. ${ }^{1}$ O presente artigo estende a análise ao caso de duas economias abertas que crescem e nas quais o crescimento fatorial

* Uma versão modificada deste artigo foi publicada, em inglês, sob o título "The Prebisch-Singer thesis and the 'widening gap' between developed and developing countries", no Canadian Journal of Economics, XVI, no 1, fevereiro de 1983, pps. 104-108. A presente tradução foi feita pelo prof. Roberto Camps de Moraes.

* * Professor Associado do Departamento de Economia da Universidade Vanderbilt.

**Agradeço a meus colegas, Samuel A. Morley e Fred M. Westfield, pelos comentários úteis feitos a uma versão anterior deste artigo.

1 Como Kemp (1969) indica, Edgeworth (1894) foi o primeiro economista a perceber a possibilidade deste fenômeno, para o qual ele usou o termo "damnification". 
e/ou o progresso técnico são tratados como exógenos. Nele, são formuladas condições para o "empobrecimento relativo" de uma dessas economias com respeito a outra. Tal empobrecimento relativo pode ser acompanhado, mas não necessariamente, pelo empobrecimento "absoluto" analisado por Johnson e Bhagwati, e, para o qual, também são apresentadas as condições para a sua ocorrência em termos per capita.

O conceito de empobrecimento relativo é claramente relevante para a discussão do "hiato crescente" entre países desenvolvidos e em desenvolvimento. ${ }^{2}$ Se, no modelo de dois países acima mencionados, o país A representa o grupo de países desenvolvidos (PDs) e o país $\mathrm{B}$ o grupo de países menos desenvolvidos (PMDs), o modelo pode ser usado para investigar o quanto os tipos de mercadorias exportadas por esses grupos de países, e as suas características de demanda e oferta, podem conduzir a um hiato crscente entre as suas rendas per capita. Este artigo também estabelece um elo entre os modelos rigorosos usados pelos teóricos do comércio internacional para analisar as implicações do crescimento econômico sobre o nível de bem estar e o trabaIho mais informal sobre as relações econômicas "Centro-Periferia" publicado trinta anos atrás por Prebisch $(1950,1959,1963)$ e Singer $(1950) .^{3}$

Em particular, o nosso modelo permitirá um exame crítico das teses de Prebisch e Singer sobre o impacto diferenciado do progresso técnico sobre o bem estar dos PDs e PMDs. Foi alegado por esses dois autores que o progresso técnico nos PDs foi retido nos mesmos sob a forma de rendas maiores, ao passo que, nos PMDs, ele foi transferido para o exterior sob a forma de

2 Ver Ward et al (1971) e a extensa bibliografia sobre as propostas de uma Nova Ordem Econômica Internacional.

3 Note-se que o modelo de crescimento e comércio de Johnson (1955) também contém um país industrial (Mancunia) que comercia com um país agrícola (Agraria). Deve-se também mencionar o trabalho de Seers (1962) sobre comparações de taxas de crescimento de produtos primários e secundários, embora o seu modelo não admita variações nos termos de intercâmbio. Findlay (1980) apresentou, recentemente, um modelo de "Norte a Sul" de economia mundial onde os termos de intercâmbio ligam entre si as taxas de crescimento das duas regiões. Contrastando com o presente artigo, Findlay supõe que as elasticidades-renda para os dois bens, em ambas as regiões, sejam unitárias, e focaliza a atenção no equilíbrio de longo prazo da economia mundial no qual Norte e Sul crescem a mesma taxa. 
preços mais baixos. A validade dessa tese tem sido questionada por vários autores, em parte devido às múltiplas explicações dadas para ela, uma das quais depende do impacto do ciclo econômico. ${ }^{4}$ Será demonstrado que essa tese referente aos benefícios diferenciados do progresso técnico corresponde a um caso especial altamente improvável.

Por outro lado, a plausibilidade de certas ordens de grandeza para as elasticidades-preço e produto da demanda pelas importações em PDs e PMDs, e para as suas taxas de crescimento fatorial e progresso técnico, sugere a verossimilhança do empobrecimento relativo (embora não do absoluto) dos PMDs. Que este resultado seja verossímil foi, indubitavelmente, percebido pelos tomadores de decisão em muitos PMDs (e racionalizado por escritores como Singer e Prebisch), e pode muito bem explicar a sua rejeição de estratégias de especialização em produtos primários baseados na doutrina das vantagens comparativas, e sua conseqüente adoção de políticas, em muitas vezes excessiva, de industrialização global.

\section{EMPOBRECIMENTO RELATIVO E ABSOLUTO.}

Essas questões serão analisadas no contexto de um modelo de comércio entre dois países com duas mercadorias, no qual o crescimento fatorial e o progresso técnico são considerados exógenos, representando os PDs o país $A$, exportador do bem 1 para o país $B$, e importador do bem 2 de $B$, re-

4 Outras explicações são baseadas na alegada prevalência de condições monopolísticas de mercado e de poderosas organizações sindicais nos PDs. Para uma avaliação da tese de Prebisch e Singer, consultar, por exemplo, Flanders (1964) e Södersten (1970, cap. 2). Note-se que a posição de Prebisch sobre a questão auto-suficiência versus livre comércio para os PMDs mudou consideravelmente quando ele se tornou Secretário-Geral da UNCTAD. Além disso, Singer (1975) "revisou" recentemente o seu artigo de 1950 e hoje acha que a sua discussão foi deficiente porque ele analisou os problemas dos PDs e dos PMD́s em termos das diferentes mercadorias que eles produzem ao invés de fazê-lo em termos (nos quais ele agora acha apropriados) dos diferentes tipos de paises que eles representam. Apesar disto, a formulação original da tese de Prebisch e Singer exerce ainda suficiente influência, especialmente nos PMDs, para merecer um exame mais rigoroso do que ela recebeu até agora. 
presentante dos PMDa. Supõe-se que os dois países permanecem incompletamente especializados nos dois bens. A demanda e a oferta de cada mercadoria em cada país depende do seu nível de produção e dos termos de intercâm. bio, $p$, definido como o preço relativo do bem 1 em termos do bem 2 (todas as variáveis sendo implicitamente definidas em termos dos seus valores no tempo $t$ ). Se $D_{i j}$ denota a demanda pelo bem i no país, $X_{i j}$ a oferta do bem $i$ no país $J$ as importações do bem $i$ e $X_{j}$ o produto total do país expresso em termos do seu bem exportável $(i=1,2 ; j=A, B)$, as demandas por importações dos países são dadas por

$$
\begin{aligned}
& M_{A}=D_{2 A}\left(X_{A}, p\right)-X_{2 A}\left(X_{A}, p\right) \\
& M_{B}=D_{1 B}\left(X_{B}, p\right)-X_{1 B}\left(X_{1 B}, p\right)
\end{aligned}
$$

O equilíbrio da balança comercial é mantido no tempo se, no tempo $t$, $M_{A}=p M_{B} \quad e$

$$
\hat{M}_{A}=\hat{p}+\hat{M}_{B}
$$

onde $\bar{z}=(\mathrm{d} z / \mathrm{dt}) / z$ é a taxa relativa de crescimento de qualquer variável $z$. Denotando por $l_{j}^{d}(P)$ a elasticidade-produto (preço) da demanda pelo bem importável no país j (todas as elasticidades sendo definidas como positivas e nenhuma mercadoria sendo inferior), e por $I_{j}^{\mathbf{S}}\left(P_{\mathbf{j}}^{\mathbf{S}}\right)$ a elasticidade-produto (preço) da oferta do bem importável no país $\mathrm{j}$, as taxas de crescimento relativo de $M_{A}$ e $M_{B}$ são, a partir de (1) e (2),

$$
\hat{M}_{A}=\frac{D_{2 A}}{M_{A}}\left(I_{A}^{d} \tilde{X}_{A}+P_{A}^{d} \hat{p}\right)-\frac{X_{2 A}}{M_{A}}\left(I_{A}^{s} \tilde{X}_{A}-P_{A}^{s} \dot{p}\right)
$$

(5)

$$
\bar{M}_{b}=\frac{D_{1} B}{M_{B}}\left(l_{B}^{D} \bar{X}_{B}-P_{B}^{d} \tilde{p}\right) \frac{X_{1 B}}{M_{B}}\left(b_{b}^{s} \bar{X}_{B}=P_{B}^{s} \tilde{p}\right)
$$

Denotando por $\mathrm{I}_{\mathrm{j}}^{\mathrm{m}}=\left(\mathrm{D}_{\mathrm{ij}} \mathrm{l}_{\mathrm{j}}^{\mathrm{d}}-\mathrm{X}_{\mathrm{ij}} \mathrm{I}_{\mathrm{j}}^{\mathrm{s}}\right) / \mathrm{M}_{\mathrm{j}}$ a elasticidade-produto da demanda pelas importações do bem $\mathrm{i}$ no país $\mathrm{j}$ e por $\mathrm{P}_{\mathrm{j}}^{\mathrm{m}}=\left(\mathrm{D}_{\mathrm{ij}} \mathrm{P} \mathrm{f}^{d}+X_{\mathrm{ij}}\right.$ 
$P_{j}^{S} / / M_{j}$ a elasticidade-preço da demanda pelas importações do bem i no país j, obtemos, a partir de (3), (4) e (5).

$$
\bar{p}=E^{-1}\left(I_{B}^{m} \hat{x}_{B}-I_{A}^{m} \hat{x}_{A}\right)
$$

onde $E$ é o "fator elasticidade" $P_{A}^{m}+P_{B}^{m}-1$, que se supõe positivo para a satisfação da condição de Marshall-Lerner. Essa expressão mostra que os termos de intercâmbio variam mais rapidamente em favor de $A$ quanto maiores forem, em B, a taxa de crescimento do produto e a elasticidadeproduto da demanda por importáveis (comparativamente a A), e quanto menor for o fator elasticidade. ${ }^{5}$

A variação na renda real de um país é dada pela variação no seu produto, medido a preços constantes e ajustado por um efeito termos de intercâmbio, igual ao produto de suas importações pela variação no seu preço relativo. Denotando po $Y_{i}$ a renda real do país $j$, expressa em termos de seu bem exportável, obtemos

$$
d Y_{A}=d X_{a}-M_{A} d(1 / p)=d X_{A}+\frac{M_{A}}{p^{2}} d p
$$

$$
d Y_{B}=d X_{B}-M_{B} d p
$$

As taxas de crescimento relativo de $Y_{A}$ e $Y_{B}$, são, conseqüentemente, dadas por

$$
\hat{\gamma}_{A}=\hat{X}_{A}+m_{A} \ddot{p}
$$

(10) $\dot{\gamma}_{B}=\ddot{X}_{B}-m_{B} \bar{p}$

onde $m_{j}$ é a propensão média a importar do país $\mathrm{j}$.

Denotando por $\hat{y}_{j}$ a taxa de crescimento relativo na renda real per capita do país j (a população de cada país tomada como sinônimo de sua força de trabalho), obtemos, de (9) e (10),

$$
\hat{y}_{A}=\hat{x}_{A}+m_{A} \ddot{p}
$$

5 Consultar Johnson (1959). 


$$
\dot{y}_{B}=\dot{x}_{B}-m_{B} \dot{p},
$$

onde $\bar{x}_{j}$ é a taxa de crescimento relativo do produto per capita do país $\mathrm{j}$, avaliado a preços constantes, e $\bar{p}$ é dado pela equação (6). Subtraindo (12) de (11) vem

$$
\bar{y}_{A}-\bar{y}_{B}=\dot{x}_{A}-\bar{x}_{B}+\left(m_{A}+m_{B}\right) \dot{p}
$$

que, se não-nulo, é uma medida do "empobrecimento relativo" ou do "hiato crescente" entre as rendas reais per capita dos dois países.

A partir de (6), (11) e (12) pode-se demonstrar que a condição para o empobrecimento absoluto de cada país (ou, inversamente, para o seu "enriquecimento"), dado que o outro país também está se expandindo, é

$$
\dot{y}_{j}<0 \text { se } M_{j} \geq \frac{\bar{x}_{j}}{\bar{x}_{j}+n_{j}} E=\frac{\bar{x}_{k}+n_{k}}{\bar{x}_{j}+n_{j}} m_{j} I_{k}^{m}
$$

onde $\mathrm{j}$ representa um dos países, $k$ o outro país $(j, k=A, B)$ e $\mu j=m_{j} I_{j}^{m}$ é a propensão marginal a importar do país j. ${ }^{6}$ A partir de (6) e (13), a condição para o empobrecimento relativo de um dos países é

$$
\bar{y}_{A}<\bar{y}_{B} \text { se } E \bar{x}_{A}-\left.\Sigma_{m}\right|_{A} ^{m}\left(\bar{x}_{A}+n_{A}\right) \gtrless E \bar{x}_{B}-\left.\Sigma_{m}\right|_{B} ^{m}\left(\bar{x}_{B}+n_{B}\right),
$$

onde $\Sigma_{m}=m_{A}+m_{B}$ é a soma das propensões médias a importar dos dois paises. $^{7}$

6 Se apenas um dos países (digamos, o país A) cresce, a expressão (14) mostra que $\vec{y}_{A}<0$ se $\mu_{A}>E \bar{x}_{A} /\left(\bar{x}_{A}+n_{A}\right)$. Esta condição difere daquela formulada por Johnson (1955), $\mu_{A}>E$, porque o nosso critério para $o$ crescimento empobrecedor é formulada em termos de per capita, o que nos parece mais apropriado. Uma ilustração gráfica das condições requeridas para um declínio na renda real global é dada por Bhagwati (1958).

7 Note-se que, se somente o país $j$ cresce e $I_{j}^{m}>0$, as expressões (14) e (15) mostram que uma vez que o empobrecimento relativo ocorre $E<\left(1+n_{j} / \bar{x}_{j}\right) \Sigma_{m} l_{j}^{m}$ enquanto que o empobrecimento absoluto ocorre se $E<\left(1+n_{j} / \dot{x}_{j}\right) m_{j} \mid l_{j}^{m}$, o primeiro resultado é mais provável do que o último. 
Para fins de ilustração das expressões (14) e (15), consideremos do is casos especiais simples. Primeiro, façamos com que $\bar{x}_{A}=\bar{x}_{B}$ de modo que o produto per capita a preços constantes dos dois países cresçam a mesma taxa. Desse modo, a equação (13) mostra que $\bar{y}_{A} \gtreqless \hat{y}_{B}$ de acordo com $\vec{p} \gtrless 0$, enquanto que a equação (14) não evita a possibilidade de empobrecimento absoluto em um país (ou, inversamente, de seu "enriquecimento").

Em segundo lugar, façamos com que a população seja constante nos dois países, de modo que $\mathrm{n}_{A}=\mathrm{n}_{B}=0$. $\mathrm{O}$ resto dessa seção será devotado a uma análise deste caso que se presta à representação gráfica. De (14) vemos que

(16) $\quad \ddot{y}_{A} \gtreqless o$ ose $m_{A} I_{B}^{m} \gtreqless\left(M_{A}-E\right) \frac{\ddot{x}_{A}}{\tilde{x}_{B}}$

Esta condição é ilustrada na figura 1, onde, para dados valores dos demais parâmetros, a razão das taxas de crescimento $\bar{x}_{A} / \bar{x}_{B}$ é representada contra o fator elasticidade $E$. $O$ ramo $C D$ da hipérbole retangular dado pelo sinal de igualdade no lado direito de (16) corresponde a $\bar{y}_{A}=0$, dado que $\mathrm{e}<\mu \mathrm{A}$. As áreas acima e abaixo deste ramo, que possui $E=\mu A$ como sua assíntota vertical, correspondem a $\vec{y}_{A}<0$ e a $\vec{y}_{A}>0$, respectivamente. $O$ intercepto vertical de $D C i_{B}^{m} / I_{A}^{m}$, excederá a unidade se a elasticidadeproduto da demanda por importações em $B$ exceder à mesma elasticidade em $A$, e vice-versa. Este é o caso suposto na construção da figura 1, já que se supõe que a elasticidade-produto da demanda por importações seja menor no país "industrializado" $A$ do que no país "agrícola" $B$. Se $E>\mu_{A} \bar{y}_{A}$ é necessariamente positivo. De (14), também decorre que

$$
\text { (17) } \bar{y}_{B} \gtreqless 0 \text { se } m_{B} I_{A}^{m} \frac{\bar{x}_{A}}{\hat{x}_{B}} \gtrless \mu_{B}-E
$$

O lugar geométrico das combinações de $\bar{x}_{A} / \ddot{x}_{B}$ e $E$ para as quais $\ddot{y}_{B}=0$ é dado pela reta $C F$, cujo intercepto vertical também é $I_{B}^{m} / I_{A}^{m}$ e cujo intercepto horizontal $\mu_{\mathrm{B}}$ pode estar à direita (como na figura 1) ou à esquerda da assíntota $E=\mu_{A}$. Além disso, as áreas acima (abaixo) dessa reta correspondem a valores dos parâmetros para os quais $\bar{y}_{B}>0\left(\bar{y}_{B}<0\right)$.

Da expressão (15) achamos que

(18) $\hat{y}_{A} \gtreqless \tilde{y}_{B}$ se $\left(E-\Sigma_{m}||_{A}^{m}\right) \frac{\bar{x}_{A}}{\bar{x}_{B}} \gtreqless E-\left.\Sigma_{m}\right|_{B} ^{m}$ 


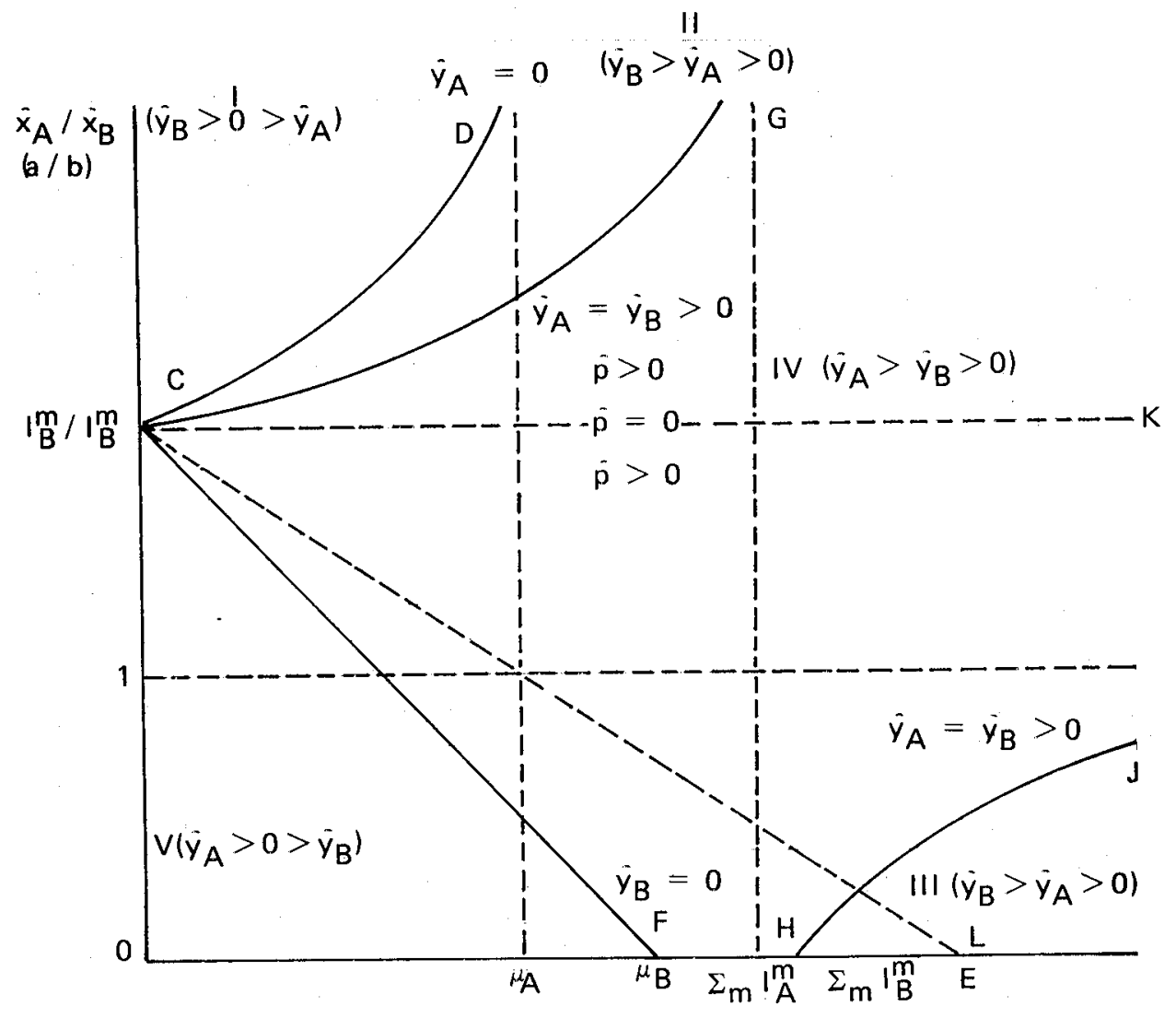

FIGURA 1

Portanto $\bar{y}_{A}=\bar{y}_{B}$ para valores de $\bar{x}_{A} / \bar{x}_{B}$ e $E$ situados nos ramos $C G$ e $H J d a$ hipérbole retangular $\bar{x}_{A} / \bar{x}_{B}=\left(E-\left.\Sigma_{m}\right|_{B} ^{m}\right) /\left(E-\Sigma_{m}||_{A}^{m}\right)$ que têm $\bar{x}_{A} / \bar{x}_{B}$ $=1$ como sua assíntota horizontal e $E=\Sigma_{m} l_{A}^{m}$ como sua assíntota vertical. Além disso, $\bar{y}_{A}>\bar{y}_{B}$ para valores dos parâmetros situados entre os dois ramos da hipérbole, enquanto que $\hat{y}_{A}<\hat{y}_{B}$ para valores situados acima do ramo CG ou abaixo do ramo $\mathrm{HJ}$.

As combinações não-negativas de $E$ e $\ddot{x}_{A} / \vec{x}_{B}$ podem ser subdivididas em cinco regiões, delimitadas na figura 1 pelos dois eixos, os ramos hiperbólicos $C D, C G$ e $H J$, e a linha $C F$, a saber, a região $I\left(\vec{\gamma}_{B}>0>\bar{y}_{A}\right)$, as regiões $I I$ e $\| I \mid\left(\bar{y}_{B}>\bar{y}_{A}>0\right)$, a região $\mid V\left(\bar{y}_{A}>\bar{y}_{B}>0\right)$ e a região $V$ $\left(\bar{y}_{A}>0>\bar{y}_{B}\right)$. As regiôes $\mid$ e $V$ correspondem ao empobrecimento absoluto 
(e relativo) de $A$ e de $B$, respectivamente, enquanto que as regiões II e III (região IV) correspondem ao empobrecimento relativo, mas não absoluto, de $A(B)$. Excetuando a linha horizontal $C K$, dada por $\ddot{x}_{A} / \ddot{x}_{B}=I_{B}^{m} / I_{A}^{m}$, para a qual $\vec{p}=0$, todas essas regiões estão associadas ou com uma deterioração, ou com uma melhoria nos termos de intercâmbio de $A$, $\rho$. Como a expressão (6) mostra, valores positivos (negativos) de $\bar{p}$ acham-se associadas a pontos abaixo (acima) de CK. Assim, nas regiões I e II, a deterioração dos termos de intercâmbio de $A$ explica o empobrecimento absoluto e relativo desse país, respectivamente, apesar do fato de que, nele, o crescimento do produto ocorre a uma taxa maior $\left(\bar{x}_{A} / \bar{x}_{B}>I_{B}^{m} / I_{A}^{m}>1\right)$. Analogamente, $o$ empobrecimento absoluto experimentado por $B$ na região $V$ é explicado, em parte, pela melhoria nos termos de intercâmbio de $A$ que mais do que compensa a maior taxa de crescimento do produ to em $B$ no caso em que $\bar{x}_{A} / \bar{x}_{B}<1$. Por outro lado, na região III, a renda real per catipa de $A$ cresce a uma taxa menor do que a de $B$ já que a melhoria nos termos de intercâmbio de $A$ não é suficiente para compensar a maior taxa de crescimento do produto de $\mathrm{B}$. $\mathrm{Fi}$ nalmente, o empobrecimento relativo de $B$ correspondente à região IV está associado à deterioração (melhoria) nos termos de intercâmbio de $A$ na subregião de IV localizada acima (abaixo) da linha CK.

A figura 1 e a análise do empobrecimento relativo e absoluto que a acompanha são baseadas no suposto de que $n_{A}=n_{B}=0$. Se as taxas de crescimento da população nos dois países são positivas, uma análise gráfica não é mais possível, mas as condições (14) e (15) mostram que, tudo o demais constante, tanto o empobrecimento relativo como o absoluto de qualquer país (e, por (6), uma deterioração dos seus termos de intercâmbio) é tão mais provável de ocorrer quanto maior for a sua taxa de crescimento demo. gráfico comparada com a do ou tro país. ${ }^{8}$

8 Pode-se verificar de (11), (12) e (6) que $\partial \hat{y}_{j} / \partial n_{j}<0, \partial \bar{y}_{j} / \partial n_{k}>0$. se $E \gtrless \Sigma_{m} l^{m} j^{\prime} \partial\left(\bar{y}_{A}-\hat{y}_{B}\right) / \partial \Sigma_{m} \gtrless 0 \gtrless 0$ e $\partial\left(\bar{y}_{A}-\bar{y}_{B}\right) / \partial E \lessgtr 0$ se $\left.\right|_{B} ^{m}$ $\left(\tilde{x}_{B}+n_{B}\right) \gtrless I_{A}^{m}\left(\bar{x}_{A}+n_{A}\right) ; \partial\left(\bar{y}_{A}-\ddot{y}_{B}\right) / \partial I_{A}^{m}<0$ e $\partial\left(\hat{y}_{A}-\bar{y}_{B}\right) / \partial I_{b}^{m}>0$. $\partial \bar{y}_{j} / \partial \ddot{x}_{j} \gtrless 0$ se $E \gtrless \mu_{j}, \partial \ddot{y}_{j} / \partial \ddot{x}_{k}>0, \partial \ddot{y}_{j} / \partial E \gtrless 0$ e $\partial \ddot{y}_{j} / \partial m_{j} \lessgtr 0$ se 


\section{A TESE DE PREBISCH E SINGER}

O modelo acima descrito pode ser usado para uma avaliação da tese de Prebisch e de Singer segundo a qual o progresso técnico nos PMDs é transferido para o exterior na forma de preços mais baixos dos produtos primários, ao passo que nos PDs ele gera rendas maiores e preços estáveis. Para isolarmos a influência do progresso técnico sobre os níveis de renda per capita, suponhamos que os estoques de fatores nos dois países permaneçam inalterados e que ocorra progresso técnico do tipo neutro de Hicks a taxas a e b em $A$ e $B$, respectivamente. Segue-se que $\bar{x}_{A}=\bar{X}_{A}=$ a e $\bar{x}_{B}=\hat{x}_{B}=b$, de modo que, na figura 1 , o eixo vertical mede agora $a / b$.

Para que o resultado de Prebisch e Singer ocorra, dado que A representa os $\mathrm{PDs}$ e $\mathrm{B}$ os $\mathrm{PMD}_{\mathrm{s}^{\prime}}$ os termos de intercâmbio $\mathrm{p}$ necessitam crescer (isto é, alterar-se em favor de A) à taxa b. Substituindo $\vec{p}=b$ em (6), obtemos

$$
a / b=\left(I I_{B}^{m}-E\right) / I_{A}^{m}
$$

Portanto, para dados valores de $\mathrm{I}_{\mathrm{A}}^{\mathrm{m}}$ e $\mathrm{I}_{\mathrm{B}}^{\mathrm{m}}$, a razão a/b teria que estar linearmente relacionada a $E$, como mostrado pela linha $C L$ na figura $1 .{ }^{9}$ Já que os valores $a, b, I_{A}^{m}, I_{B}^{m}, P_{A}^{m}, P_{B}^{m}(e$, portanto $E$ ) são, todos, independentes entre si, o resultado de Prebisch e Singer é, claramente, um caso especialíssimo e improvável de ser observado na prática. Além disso, é interessante notar que, na figura 1, mesmo que os valores de a/b e $E$ estivessem situados na linha $C L$, este resultado não seria consistente com o crescimento empobrecedor no país $B$, ainda que se presumisse que esse país estivesse "transferindo" para o exterior os benefícios do seu progresso técnico. ${ }^{10}$

$i_{j}^{m}\left(\bar{x}_{j}+n_{j}\right) \gtrless i_{k}^{m}\left(\bar{x}_{k}+n_{k}\right), \partial \bar{y}_{j} / \partial I_{j}^{m}<0$ e $\partial \bar{y}_{j} / \partial I_{k}^{M}>0$, onde j repre senta um dos países e $\mathrm{k}$ o outro. Além disso, (6) e (13) mostram que $\partial\left(\dot{y}_{A}-\bar{y}_{B}\right) / \partial \bar{n}_{B}>0, \partial\left(\bar{y}_{A}-y_{B}\right) / \partial n_{A}<0, \partial\left(\dot{y}_{j}-\dot{y}_{k}\right) / \partial \dot{x}_{j} \gtrless 0$

9 O intercepto horizontal $L$ de $C L$ é igual a $l_{\mathrm{B}}^{\mathrm{m}} \mathrm{e}$, portanto, situa-se à direjta ou à esquerda de $H$, dependendo de se $\Sigma_{m}$ é menor ou maior do que 1 . ${ }_{10}$ A razão para isto é que o intercepto horizontal de $C L, I_{B}^{m}$, é necessaria- 
Embora a tese de Prebisch e Singer, na sua forma extrema, seja alta. mente implausivel, a figura 1 esclarece algo sobre as perspectivas de um "hiato crescente" entre os PDs e os PMDs. Adotando as hipóteses plausíveis de que as exportações dos PMDs possuam uma elasticidade-produto da demanda menor do que as dos PDs $\left(1_{B}^{m} / I_{A}^{m}>1\right.$, como mostrado no diagrama) e de que a taxa de progresso técnico nos PDs exceda à dos PMDs $(a / b>1)$, os valores de $a / b$ e $E$ cairiam na sub-região da região IV que se situa acima da assíntota horizontal $a / b=1$, mesmo para valores moderadamente baixos do fator elasticidade E. ${ }^{11}$ Isto implicaria um "hiato crescente" entre os PDs e os PMDs no sentido de que o crescimento da renda real per capita nos PMDs, embora positivo, seria menor do que o observado nos PDs. Este resultado qualitativo vigoraria (não, obviamente, o valor particular da taxa de empobrecimento relativo de $B$ ) independentemente de se os termos de intercâmbio variassem em favor de $A$ (como ocorreria se $a / b<1 \mathrm{~B}_{\mathrm{B}}$ / $\left(m_{A}^{m}\right.$ ) ou contra $A$ (para $\left.a / b>1 m_{B}^{m} / / m_{A}^{m}\right)$. Vê-se, portanto, que os movimentos nos termos de intercâmbio são uma medida inadequada das variaç̃̃es no bem estar, sendo o seu papel suplementar. Essas conclusões seriam reforçadas se, como também é plausível, o crescimento fatorial causasse um crescimento mais rápido do produto per capita em $A$ do que em $B$, de modo que $\ddot{x}_{A}$ ' $\hat{\mathrm{x}}_{\mathrm{B}}>\mathrm{a} / \mathrm{b}>1 .{ }^{12}$

mente maior do que o de $C F$, dado por $\mu_{B}=m_{B}||_{B}^{m}$. Note-se que, sob condições competitivas, os termos de intercâmbio são determinados pela demanda, dadas ofertas exógenas nos dois países, de modo que o modelo não comporta diferenças nas estruturas dos mercados de trabalho entre os países industrializados e os agrícolas. Por sua vez, Singer (1950), depois de afirmar que os termos de intercâmbio deterioram-se para os países agrícolas devido à forma que o progresso técnico assume neles (isto é, preços menores ao invés de salários maiores), alega que as condições de demanda: são uma causa independente da deterioração dos termos de intercâmbio. Assim, depois de discutir a assimetria na distribuição dos frutos do progresso técnico, ele escreve: "Outros fatores (condições de demanda) também contribuíram para a tendência de declínio de longo prazo dos preços dos produtos primários em termos de manufaturados, além da ausência de pressão dos produtores por maiores rendas." (p. 479, a ênfase é minha).

11 Este resultado seria assegurado se $E>\left.\Sigma_{m}\right|_{A} ^{m}$.

${ }^{12}$ Como a nota 8 mostra, isto também está sujeito à condição $E>\Sigma_{m} \mid{ }_{A}^{m}$. 


\section{CONCLUSŌES}

A resposta à questão de se o crescimento produz uma piora absoluta no bem estar de um país depende do valor da taxa de crescimento no seu produto per capita a preços constantes relativamente ao produto de sua propensão média a importar vezes a taxa de deterioração de seus termos de intercâmbio. A taxa de variação nos termos de intercâmbio desempenha um papel ainda mais importante na determinação de se um país melhora ou piora o seu bem estar em relação ao seu parceiro comercial, já que o ganho na renda real per capita de um país oriundo de uma variação nesse índice é necessariamente correspondido (embora não igual em valor absoluto, dado que $m_{A} \neq m_{B}$ ) por uma perda no outro país. Foram formuladas condições para o crescimento absoluta e relativamente emprobrecedor em um mundo com dois países, condições essas derivadas em termos de elasticidades-preço e produto das demandas e ofertas de bens importáveis e das taxas de crescimento demográfico e do produto per capita dos dois países.

Como um subproduto desta análise, foi demonstrado que a tese de Prebisch e Singer referente ao impacto diferenciado do progresso técnico nos PDs e PMDs é, em geral, incorreta. Entretanto, pode-se esperar que maiores elasticidades-produto na demanda por importações nos PMDs combinadas com taxas mais baixas de progresso técnico e taxas mais altas de crescimento populacional devem conduzir a um hiato crescente entre as rendas reais per capita dos PDs e dos PMDs. Isto sugere, tal como no caso do argumento protecionista baseado na indústria nascente, que existam vantagens dinâmicas de longo prazo derivadas de uma política de industrialização nos PMDs, embora ao custo de curto prazo de menores ganhos de comércio nos produtos tradicionalmente exportados por esses países.

\section{BIBLIOGRAFIA}

Bhagwati, J. (1958), "Immiserizing Growth: A Geometrical Note," Review of Economic Studies, vol. 25, pp. 201-205.

Edgeworth, F. Y. (1894), "The Theory of International Values. I," Économic Journal, vol. 4, no. L, pp. 35-50. 
Findlay, R. (1980), "The Terms of Trade and Equilibrium Growth in the World Economy", American Economic Review, vol. 70, no 3, pp. 291-299.

Flanders, M. J. (1964), "Prebisch on Protectionism: An Evaluation," Economic Journal, vol. 74, pp. 305-326.

Johnson, H. G. (1953), "Equilibrium Growth in an International Economy," The Canadian Journal of Economics and Political Science, vol. 19, n० 4, pp. 478-500.

(1955) "Economic Expansion and International Trade,"

The Manchester School of Economic and Social Studies, vol. 23, no 2, pp. 95-112.

(1959), "Economic Development and International Trade,"

reprinted in H. G. Johnson, Money, Trade and Economic Growth, Harvard University Press, Cambridge, 1962, pp. 75-103.

Kemp, M. C. (1969). The Pure Theory of International Trade and Investment, Prentice-Hall, Englewood Cliffs, N. J.

Prebisch, R. (1950), The Economic Development of Latin America and Its Principal Problems, U. N. Economic Commission for Latin America, New York.

(1959), "Commercial Policy in the Underdeveloped Coutries, "Ame-

rican Economic Review, Papers and Proceedings, Vol. 49, pp. 251273.

(1963), Towards a Dynamic Development Policy for Latin

America, United Nations, New York.

Seers, D. (1962), "A Model of Comparative Rates of Growth in the World Economy, “Economic Journal, vol. 72, pp. 45-78.

Singer, H. W. (1950), "The Distribution of Gains Between Investing and Borrowing Countries, "American Economic Review, Papers and Proceedings, Vol. 40, pp. 473-485.

(1975), "The Distribution of Gains from Trade and Invest-

ment - Revisited, “Journal of Development Studies, vol. 11, no 4, pp. 376-382.

Södersten, B. (1970), International Economics, Harper and Row, New York.

Ward, B. et al. (eds.) (1971), The Widening Gap: Development in the 1970's, Columbia University Press, New York. 